Meta

Journal des traducteurs

Translators' Journal

\title{
Conversation avec Patricia Claxton (présidente de l'Association des traducteurs littéraires)
}

\section{Pierre Marchand}

Volume 22, numéro 1, mars 1977

Histoire de la traduction au Canada

URI : https://id.erudit.org/iderudit/003556ar

DOI : https://doi.org/10.7202/003556ar

Aller au sommaire du numéro

Éditeur(s)

Les Presses de l'Université de Montréal

ISSN

0026-0452 (imprimé)

1492-1421 (numérique)

Découvrir la revue

Citer ce document

Marchand, P. (1977). Conversation avec Patricia Claxton (présidente de

l'Association des traducteurs littéraires). Meta, 22(1), 79-87.

https://doi.org/10.7202/003556ar d'utilisation que vous pouvez consulter en ligne. 


\title{
Conversation avec Patricia Claxton
}

\author{
présidente de l'Association des traducteurs littéraires
}

\section{Le marché de la traduction}

META - Si vous le voulez bien, j'aimerais d'abord que vous nous renseigniez un peu sur la situation du traducteur littéraire au Québec et au Canada. Plus particulièrement, comment peut-on décrire le marché de la traduction littéraire et les relations entre les traducteurs et les maisons d'édition?

P. C. - Les traducteurs littéraires sont tous pigistes; ils signent donc un contrat chaque fois qu'ils traduisent un livre. Quant à la rémunération du traducteur, elle se rattache à la question des subventions accordées par l'État.

En pratique, actuellement, tous les travaux de traduction littéraire sont effectués sous l'égide du Conseil des arts du Canada, qui accorde des subventions aux éditeurs. Ces subventions servent à favoriser l'édition d'œuvres traduites. Les cuvres originales doivent être canadiennes, de même que les traducteurs, et les langues de départ et d'arrivée doivent être l'anglais ou le français.

D'autre part, le Secrétariat d'État subventionne, à l'occasion, la traduction, en anglais ou en français, d'ouvrages écrits par des Canadiens et dans une langue autre que l'anglais ou le français. Rien n'interdirait, d'autre part, l'octroi de subventions pour la traduction, en langue étrangère, d'ouvrages canadiens rédigés en anglais ou en français. Il ne s'agit pas cependant d'un véritable « programme ». Il se trouve tout simplement qu'il y a des fonds disponibles et qui peuvent être utilisés pour l'octroi des subventions dont je viens de parler.

META - Qui doit demander la subvention au Conseil des arts : le traducteur ou l'éditeur?

P. C. - C'est l'éditeur qui demande la subvention et c'est à l'éditeur que la subvention est accordée. La somme octroyée sert alors à rémunérer le traducteur, éventuellement à payer certains frais connexes, par exemple pour des révisions importantes.

META - Quelle est, justement, la rémunération normalement accordée au traducteur?

P. C. - Le maximum accordé par le Conseil des arts est de 5 cents le mot et c'est, dans la plupart des cas, le maximum que reçoit le traducteur. Les éditeurs 
demandent invariablement le maximum autorisé, ce qui ne veut pas forcément dire que le traducteur a toujours reçu la totalité de la subvention accordée. Car certains éditeurs se sont crus en droit d'effectuer des prélèvements afin de se défrayer des sommes imputables à l'édition de l'œuvre traduite. C'est pourquoi les traducteurs ont demandé au Conseil des arts de s'assurer que les subventions soient intégralement versées aux traducteurs et qu'elles ne servent pas, en partie, à payer les frais de production. À côté du prix à forfait, on voit parfois des formules à base de droits d'auteur (appelés tantièmes), mais elles ne changent pas grand-chose en général.

META - À raison d'une rémunśration maximale de 5 cents le mot, est-ce que vous estimez que l'on peut en 1976, vivre de la traduction littéraire?

P. C. - Si l'on pouvait avoir des textes relativement simples, peut-être. Mais les ouvrages à traduire sont souvent difficiles et, en réalité, le traducteur littéraire n'arrive pas à joindre les deux bouts. Je dois ajouter que, dans quelques cas, la rémunération du traducteur a été supérieure à 5 cents le mot. Il s'est agi, dans ces cas, d'un supplément versé par l'éditeur lui-même.

META - Normalement, donc, l'éditeur ne participe pas financièrement au travail de traduction proprement dit.

P. C. - En pratique, très peu d'éditeurs contribuent financièrement à la traduction des ouvrages. Mais si les traducteurs sont payés assez modestement, on nous répète qu'ils gagnent quand même plus que les auteurs qu'ils trađuisent.

META - Estimez-vous que les traducteurs sont trop bien payés ou que ce sont les auteurs qui ont un rattrapage à faire?

P. C. - C'est la seconde proposition qui m'apparaît la plus juste...

META - Il existe une Convention universelle sur le droit d'auteur. Y a-t-il des pays qui appliquent cette convention aux traducteurs?

P. C. - Les pratiques varient beaucoup d'un pays à l'autre. Il faut d'abord reconnaître que, sur le plan de la rémunération, les traducteurs canadiens sont mieux traités que dans bien d'autres pays. Nous ne connaissons pas la situation dans chacun des pays, mais nous pouvons dire qu'en ce qui concerne les pays avec lesquels nous avons les contacts les plus fréquents, il est difficile de prétendre que les traducteurs qui s'y trouvent sont mieux traités qu'ici.

META - Est-ce que cela voudrait dire que si la Convention universelle sur le droit d'auteur s'appliquait aux traducteurs canadiens, cela ne changerait pas grand-chose à la situation ?

P. C. - Il faut d'abord signaler que les tirages, même en langue d'origine, ne sont pas très élevés. Au Canada anglais, on considère qu'un tirage à 2000 est un succès et que la chose est peut-être même exceptionnelle. On voit donc que l'obtention de droits d'auteur, par tous les traducteurs, ne changerait pas grand-chose. Mais nous essayons quand même de faire reconnaître le principe, car si un ouvrage traduit connaît un succès de vente spectaculaire, il nous semble rien 
moins que juste que le traducteur retire une certaine part des bénéfices que son travail a fait réaliser. Il me semble en effet tout à fait raisonnable de supposer que le succès de ce livre est dû, en grande partie, au travail du traducteur.

META - Comme les subventions du Conseil des arts ne sont accordées que pour la traduction d'ouvrages canadiens, faut-il en conclure que les traducteurs, pour la majorité, traduisent des ouvrages canadiens et ne s'occupent pas des ouvrages publiés ailleurs qu'au Canada?

P. C. - C'est en effet le cas; et il faut ajouter que, de toute façon, la rémunération en provenance de l'étranger ne serait pas très élevée. Il faut penser qu'en France, par exemple, on verse l'équivalent de 2 cents le mot, et moins.

META - Est-ce la raison pour laquelle les ouvrages américains, pour ne prendre qu'un exemple, sont surtout traduits en France ?

P. C. - Oui. Et puis on vise le marché européen. Et même aux États-Unis, la rémunération est d'environ 2,5 cents le mot et, au maximum, de 3 cents.

META - Mais, est-ce que vous ne pensez pas que les éditeurs canadiens, comme les traducteurs, auraient intérêt à faire traduire et à éditer eux-mêmes les ouvrages en provenance de l'étranger? Ne serait-il pas avantageux, également, qu'un éditeur québécois puisse faire traduire ici des ouvrages américains en vue de les distribuer dans les pays francophones?

P. C. - En effet, il serait raisonnable de le supposer, mais on entre là dans le domaine de l'édition et des relations entre les éditeurs. Ceux-ci, comme on le sait, sont assez jaloux de leur propre production. Par exemple, je vois difficilement comment un éditeur français accepterait qu'un de ses auteurs soit publié en langue anglaise au Canada avant de l'être aux États-Unis, où le marché est beaucoup plus grand. Si un éditeur canadien arrivait à vendre aux États-Unis un ouvrage qu'il a lui-même fait traduire, tant mieux. Mais il est vraiment très difficile d'y parvenir, sauf pour des ouvrages canadiens. On voit donc que tout le problème se ramène à celui de l'édition et de la rentabilité de la traduction.

META - Cela m'amène à faire une comparaison entre la traduction des livres et le doublage des films. On sait que, depuis quelques années, la société Radio-Canada exerce des pressions auprès des producteurs étrangers pour que ceux-ci fassent doubler au Canada un nombre déterminé de films destinés à la télévision. Cette mesure a été prise afin d'encourager les artistes d'ici de même que l'industrie canadienne du doublage. Est-ce que l'on ne pourrait pas imaginer une solution semblable dans le domaine de la traduction littéraire?

P.C. - Je pense que les éditeurs étrangers se fichent pas mal du marché canadien. L'Etat fait ce qu'il peut pour aider l'édition dans tous les sens possibles, mais les avis sont très partagés sur les meilleurs moyens d'y parvenir. La situation de l'édition au Canada, du côté anglais comme du côté français, n'est pas facile.

META - Il semble, cependant, que les producteurs américains de cinéma aient accepté de faire doubler ici certains de leurs films ; ils ont dû se dire, par conséquent, que le marché canadien en valait la peine. Pourquoi ne serait-ce pas 
le cas en ce qui concerne l'édition, et je songe ici à l'adaptation des ouvrages didactiques, particulièrement ceux qui sont destinés au marché francophone du Québec?

P. C. - Ce qui se produit, en fait, c'est que presque tous les ouvrages scolaires anglais traduits en français pour le Québec, le sont déjà ici. Mais ce qui est intéressant, c'est que l'on comminence à constater une certaine insistance sur l'étude d'ouvrages canadiens; partout au Canada on se rend compte du très grand intérêt manifesté envers les littératires canadiennes tant en français qu'en anglais. Cette situation pourrait aussi favoriser la traduction des ouvrages publiés sur la question.

Il y a aussi des ouvrages savants, sur l'histoire, la sociologie, l'économique, l'art, qui ne sont pas des manuels scolaires, mais qui sont destinés au milieu universitaire et au grand public. Ces ouvrages ont conmencé à être traduits, et le mouvement va certainement se continuer.

META - Cela signifie, par conséquent, que la situation du traducteur, au Canada et au Québec, est étroitement liée aux mouvements d'idées qui se manifestent chez nous. Peut-être ne s'est-on pas suffisamment arrêté sur cette question.

P. C. - Cela est vrai ; il faut d'autre part reconnaître que c'est grâce au Conseil des arts que les idées peuvent être ainsi véhiculées par la traduction. Car le Conseil des arts ne subventionne pas que la traduction d'ouvrages littéraires; il s'intéresse aussi aux ouvrages savants, à l'exception des ouvrages scolaires proprement dits, puisque l'on considère que ceux-ci sont déjà rentables.

META - Nous avons parlé du Conseil des arts, mais j'aimerais savoir si d'autres organismes s'occupent de favoriser la traduction d'ouvrages rédigés ici.

P.C. - Oui, le Conseil des arts de l'Ontario a déjà subventionné des travaux de traduction. On espère qu'il en sera de même du Québec, où le ministère des Affaires culturelles vient d'annoncer la création du Conseil de la culture. Mais j'ai un peu l'impression que les préoccupations de ce nouvel organisme concerneront, au début du moins, la création d'ouvrages plutôt que la traduction.

META - Mais, actuellement, le gouvernement québécois ne subventionne pas la traduction?

P.C. - Pas que je sache. Je crois que le gouvernement du Québec a prévu la chose, mais jusqu'à maintenant, à ma connaissance, on n'a pas accordé de subventions à la traduction. On sait cependant qu'il subventionne la Saberdache québécoise, pour l'établissement d'une liste d'ouvrages québécois susceptibles d'être traduits. Malheureusement, la liste ne comprendra pas les ouvres de création.

\section{Les relations professionnelles entre le traducteur et l'éditeur}

P. C. - J'aimerais, si vous le voulez bien, attirer votre attention sur un aspect particulier des relations entre les traducteurs et les éditeurs.

Les éditeurs, surtout ceux de langue anglaise, commencent seulement à comprendre ce qu'est une bonne traduction. Les éditeurs francophones, par la 
force des choses probablement, sont déjà un peu plus évolués : leurs lecteursréviseurs connaissent bien la langue de départ et ils ont souvent traduit euxmêmes ; ils sont par conséquent capables de juger la qualité d'une traduction.

Mais, du côté anglais, la situation est bien différente. Les traducteurs vers l'anglais ont souvent à discuter avec un lecteur-réviseur qui ne comprend pas un seul mot de la langue de départ. Et ces réviseurs font des changements dans nos textes, sans l'autorisation du traducteur. C'est une chose dont les traducteurs anglais se plaignent beaucoup. Moi-même, j'ai eu à me bagarrer souvent avec les lecteurs-réviseurs.

Nous essayons actuellement d'obtenir le droit de regard sur le texte définitif. L'Association des traducteurs littéraires (A.T.L.A.) met au point, en ce moment, un projet d'entente type qui, entre autres choses, prévoira justement cette question de la révision et qui veillera à faire préserver l'intégrité du travail du traducteur ; c'est en effet le traducteur qui doit être responsable de son travail. Il arrive bien sûr qu'un éditeur puisse rejeter une traduction totalement inacceptable, mais il y a aussi des cas où d'excellents traducteurs ont eu beaucoup de difficultés avec leur éditeur. L'A.T.L.A. se propose de travailler avec les éditeurs pour établir un mécanisme d'appel en cas de différend sur ce point.

META - Si les anglophones sont moins conscients que les francophones de la difficulté de traduire, c'est peut-être qu'ils ont eu moins l'occasion de réfléchir à la question, étant donné que le phénomène de la traduction, dans son ensemble, c'est surtout le Québec qui le connaît.

P. C. - Il est certain qu'au Québec, les consommateurs de traduction sont davantage sensibilisés à la question. Mais j'ajouterai que, depuis un an et demi, les traducteurs littéraires constatent un certain progrès de la part des éditeurs, qui comprennent mieux les problèmes en cause ; c'est déjà beaucoup.

\section{Le point sur l'Association des traducteurs littéraires}

META - Il existe, on le sait, une Association des traducteurs littéraires ${ }^{1}$. Est-ce que vous pensez qu'elle a joué un rôle dans l'avancement de la question de la traduction littéraire au Canada, particulièrement en ce qui concerne les progrès dont vous venez de parler?

P. C. - Oh ! certainement! Les éditeurs savent que l'Association existe et que tous les traducteurs importants, dans le domaine littéraire, en font partie. En fait la plupart des traducteurs littéraires sont membres de l'Association (nous sommes actuellement une quarantaine). C'est donc dire que les prises de position de l'A.T.L.A. ont maintenant beaucoup de poids. Les progrès sont également attribuables aux subventions du Conseil des arts et à l'institution du Prix de traduction.

1. Voir à ce sujet l'article de Jean-Paul Partensky, * Fondation de l'Association des traducteurs littéraires * dans Meta, vol. 20, no 3, p. 244 et 245. 
META - Quels sont les moyens qu'a l'intention de prendre l'Association pour parvenir à l'un de ses objectifs, qui est l'amélioration de la qualité de la traduction littéraire?

P. C. - Il y a l'entente type, dont je vous ai un peu parlé tout à l'heure. Mais pour améliorer la qualité de la traduction, nous ne prévoyons pas, par exemple, donner des cours de perfectionnement. Ce que nous pouvons faire, cependant, c'est de fournir aux traducteurs l'occasion d'échanger leurs points de vue, de se consulter au besoin, etc. Cela est très important, car les traducteurs littéraires travaillent seuls. Il leur est parfois difficile de garder le contact avec les autres traducteurs, à moins de pouvoir le faire par le truchement d'une association.

META - Est-ce que votre association a des relations avec les éditeurs ?

P. C. - Jusqu'à maintenant, nous avons eu surtout des relations non officielles. Ce que nous avons fait qui soit officiel, ce fut de demander au Conseil des arts de préciser sa politique de subventions et d'indiquer très précisément à chacun des éditeurs le véritable pourquoi des subventions ; nous avons également demandé au Conseil des arts de communiquer au traducteur la somme remise à l'éditeur à titre de subvention à la traduction. Très souvent, dans le passé, le traducteur n'a pas su quelle somme recevait son éditeur.

META - Et quelle a été la réponse du Conseil des arts ?

P. C. - La réponse du Conseil des arts a été favorable. Par conséquent, chaque traducteur doit dorénavant recevoir un avis de l'octroi de la subvention qui l'intéresse.

META - Revenons, si vous le voulez bien, à la question de la qualité du travail. Pour être admis à l'Association des traducteurs littéraires, on n'a pas à justifier de sa compétence; il suffit d'avoir traduit un livre ou l'équivalent. $\mathrm{Ne}$ croyez-vous pas que ce critère d'admission, la qualité du travail, manque à votre association?

P. C. - Non, car il nous a semblé très dangereux d'essayer de juger le travail de nos membres; il s'agit pour nous de regrouper ceux qui travaillent dans le domaine ; le jugement de la qualité, en traduction littéraire, est encore plus subjectif que dans les autres formes de traduction. On risquerait par conséquent de former des cliques et on a voulu éviter cela. Nous considérons que c'est par « attrition », peut-être, qu'on devrait parvenir à une étape où tous les membres seront d'excellents traducteurs. Peut-être devrais-je retirer le mot « attrition », il pourrait être mal compris ; en fait, c'est surtout en travaillant à la reconnaissance de l'excellence en traduction littéraire et en encourageant les traducteurs, dont la compétence est reconnue, à s'associer à nous, que nous parviendrons le plus efficacement à faire accéder les traductions littéraires au statut d'œuvres d'art.

META - On peut également compter sur le fait que les traducteurs littéraires ne travaillent pas dans l'anonymat ; cela facilite peut-être la communication entre traducteurs. 
P. C. - Oui, et nous demandons justement que le nom du traducteur apparaisse sur la couverture du livre; nous demandons en fait que le traducteur soit traité de la même façon que l'auteur en ce qui concerne les renseignements bibliographiques, la publicité, et le reste. Nous voulons que le traducteur soit associé à son travail.

Nous considérons également, et là-dessus nous rejoignons la S.T.Q., qu'il est hautement désirable que le nom du traducteur soit toujours connu, même lorsqu'il ne s'agit pas de traduction littéraire.

META - En effet, l'anonymat est peut-être, trop souvent, un peu commode. On peut se demander si certaines traductions pitoyables, que l'on a vu circuler, auraient été telles si les traducteurs avaient su dès le départ que leur nom allait être publié. Ce que certains considèrent comme un privilège - la publication du nom de l'auteur - est en fait une responsabilité.

P. C. - Justement, j'ai vu qu'au Québec on commençait à publier le nom du traducteur, dans certaines publications de nature très technique. Je l'ai vu en tout cas dans certaines brochures de l'Hydro-Québec. Je suis persuadée que c'est là un des moyens les plus efficaces d'améliorer la qualité de la traduction.

META - Mais par quel moyen peut-on réussir à faire publier le nom des traducteurs : en exerçant des pressions auprès des éditeurs, en ayant recours au pouvoir de l'État...?

P. C. - Nous comptons faire mettre la chose dans notre modèle de contrat.

META - Cela va donc se décider au cours de chacune des négociations entre le traducteur et l'éditeur?

P. C. - Oui, car tous les rapports s'établissent entre les traducteurs et les éditeurs, ou les producteurs de films, etc. Il nous semble beaucoup plus direct d'y arriver par la voie de l'entente. Il me semble d'ailleurs que les éditeurs ne sont pas contre la chose ; c'est, tout simplement, qu'ils n'y ont jamais pensé.

META - En effet, on ne voit pas pourquoi les éditeurs s'y opposeraient, car cela ne leur ferait aucun tort.

P. C. - Et dans certains cas, lorsque le traducteur est avantageusement connu, cela pourrait leur être profitable.

META - Voudriez-vous nous parler maintenant des relations entre 1'Association des traducteurs littéraires et les autres associations de traducteurs?

P. C. - L'A.T.L.A. a été fondée à la demande des traducteurs littéraires eux-mêmes. Ceux-ci n'ont pas jugé qu'une association avec les grands groupements de traducteurs pourrait les aider à régler leurs problèmes, qui sont particuliers. Je dois d'autre part signaler que certains des membres de l'Association sont également membres de la S.T.Q. et ne voient pas de contradiction dans cette double appartenance. 
META - Mais est-ce que cela ne vous prive pas de certaines relations avec les associations étrangères qui, elles, regroupent des traducteurs littéraires ? On sait en effet qu'il faut passer par le Conseil des traducteurs et interprètes du Canada (C.T.I.C.) pour avoir des relations officielles avec les sociétés membres de la Fédération internationale des traducteurs (F.I.T.), et que pour être membre du C.T.I.C., il faut être une société provinciale.

P. C. - Cela nous prive certainement de certaines relations, surtout lorsqu'il s'agit de participer aux congrès internationaux. Nous sommes entrés en contact avec des groupements étrangers, mais nous considérons important, par exemple, que l'A.T.L.A. soit présente au prochain congrès de la F.I.T.; pour ce faire, nous aimerions nous associer, d'une façon ou d'une autre, avec le C.T.I.C., car nous ne voulons pas agir séparément; nous préférons faire partie de la représentation canadienne dans son ensemble.

Mais les membres de l'A.T.L.A. ont été catégoriques sur un point : s’il est impossible de passer par le C.T.I.C. pour ce qui est de notre représentation internationale, nous serons prêts à rechercher une représentation directe, au sein de la F.I.T. Mais nous souhaitons ne pas avoir à prendre cette décision. Nous espérons trouver, conjointement avec le C.T.I.C., une solution à l'obstacle créé par le fait que l'Association des traducteurs littéraires n'est pas une société provinciale.

META - Si vous avez jugé bon de fonder une société distincte, est-ce que cela veut dire que les sociétés membres du C.T.I.C. ne se sont pas suffisamment intéressées à la question de la traduction littéraire?

P. C. - Cela a certainement été le cas, jusqu'à maintenant, et c'est l'une des raisons pour lesquelkes les traducteurs littéraires se sont regroupés.

META - Maintenant, est-ce que vous prévoyez que l'Association des traducteurs littéraires vivra indéfiniment ou est-ce qu'elle finira par fusionner avec le C.T.I.C. ou avec chacune des sociétés membres du C.T.I.C.?

P. C. - Tout est possible, bien sûr, mais je n'oserais pas en dire davantage.

META - J'ai l'impression qu'il fallait que votre Association naisse pour que les autres sociétés se rendent compte de l'existence des traducteurs littéraires.

P. C. - Le fait est qu'en littérature, l'activité traduisante se rapproche beaucoup plus de l'activité écrivante que de la traduction proprement dite. Il y a d'abord le fait que le travail du traducteur littéraire se vend alors que ce n'est pas exactement la même chose en ce qui concerne les autres traducteurs. Et nous devons négocier directement et constamment avec les éditeurs; c'est donc dire que nous sommes seuls. De plus, nous devons souvent nous occuper nous-mêmes des questions de mise en page, de présentation typographique. Et les textes que nous avons à traduire s'éloignent souvent de la façon courante d'écrire ; cela change considérablement le travail du traducteur.

META - Cela a pu expliquer votre besoin de vous regrouper ; et peut-être, d'autre part, que les traducteurs \& non littéraires \$ n'ont pas tellement compris votre situation. 
P.C. - C'est un autre aspect, également : le traducteur littéraire gagne beaucoup moins que les traducteurs dans l'entreprise. Les traducteurs littéraires sont peut-être considérés comme un peu fous. En fait, ils aiment tellement leur métier qu'ils sont prêts à sacrifier des revenus plus importants.

META - Il nous faut espérer que la création de votre Association amènera tous les autres traducteurs, et les sociétés qui les regroupent, à prendre conscience de la situation du traducteur littéraire. Et si j’avais un souhait à formuler, ce serait de voir l'Association des traducteurs littéraires revenir aux sociétés membres du C.T.I.C. lorsque cette prise de conscience sera pleinement réalisée.

P. C. - Personnellement, je le souhaite aussi, car je crois beaucoup à la nécessité des associations d'envergure.

Propos recueillis par Pierre Marchand 